\title{
Giant Molecular Cloud Populations in Nearby Galaxies
}

\author{
Annie Hughes ${ }^{1}$, Sharon Meidt ${ }^{2}$, Dario Colombo ${ }^{3}$, Andreas Schruba ${ }^{4}$, \\ Eva Schinnerer ${ }^{2}$, Adam Leroy ${ }^{5}$, Tony Wong $^{6}$ and the PAWS, \\ MAGMA, CANON and M31-CARMA survey teams \\ ${ }^{1}$ Institute for Research in Astrophysics \& Planetology, CNRS \\ email: Annie.Hughes@irap.omp.eu \\ ${ }^{2}$ Max Planck Institute for Astronomy \\ ${ }^{3}$ Department of Physics, University of Alberta \\ ${ }^{4}$ Max Planck Institute for Extraterrestrial Physics \\ ${ }^{5}$ Department of Astronomy, The Ohio State University \\ ${ }^{6}$ Astronomy Department, University of Illinois
}

\begin{abstract}
We present new results from a comparative analysis of the resolved giant molecular cloud (GMC) populations in six nearby galaxies. We show that the GMCs in denser environments - M51, the centre of NGC6946 - have greater CO surface brightness and higher velocity dispersions relative to their size than GMCs in less dense environments. We find systematic differences in the GMC mass distribution among galaxies, such that more of the molecular gas in the low-mass galaxies (M33, the Large Magellanic Cloud) and the outer disk of M31 is located in low mass clouds. Using the number density of GMCs in the interarm regions of M51, we argue that GMC destruction in this region is regulated by shear, and that cloud lifetimes there are finite and short, $\sim 20$ to $30 \mathrm{Myr}$. Our results indicate the importance of galactic environment on the evolution of GMCs, and on a galaxy's global pattern of star formation.
\end{abstract}

Keywords. ISM: clouds, galaxies: ISM, (galaxies:) Magellanic Clouds, (galaxies:) Local Group

\section{Introduction}

Most star formation occurs within giant molecular clouds (GMCs): cold, dense ( $T \sim$ $10 \mathrm{~K}, n \sim 10^{3} \mathrm{~cm}^{-3}$ ) structures with a typical mass of $\sim 10^{5} \mathrm{M}_{\odot}$ and a characteristic size of $\sim 40$ pc. Studies of the Milky Way's GMC population have shown that they are in approximate virial equilibrium, with scaling relations between their size, velocity dispersion and CO luminosity (e.g. Solomon et al. 1987; henceforth S87). Investigating GMCs in external galaxies presents the opportunity to learn how the distribution, density structure and dynamical state of star-forming clouds depends on galactic environment, and how galactic-scale processes influence GMC formation and destruction. Until recently, however, the observations required to resolve extragalactic GMCs were technically demanding. In this contribution, we compare the properties of GMCs identified in wide-field, high resolution CO surveys of six galaxies: M51 (Pety et al. 2013; Schinnerer et al. 2013), NGC6946 (Donovan Meyer et al. 2013), NGC628 (Schinnerer et al., in prep.), M31 (Schruba et al., in prep.), M33 (Rosolowsky et al. 2007) and the Large Magellanic Cloud (LMC, Wong et al. 2011). We highlight results that connect the properties and timescales of individual clouds to their galactic context. The results in Sections 1 and 2 are presented in Hughes et al. (in prep.); the results in Section 3 are presented in Meidt et al. 2015. 

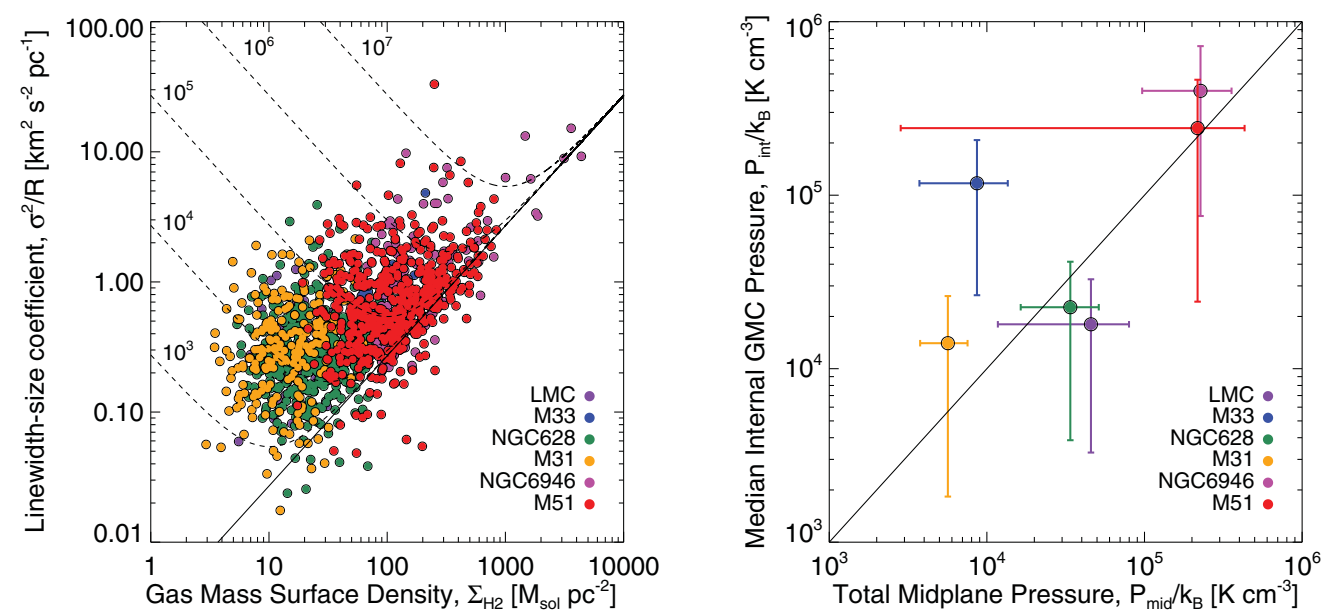

Figure 1. Left. The coefficient of the linewidth-size relation, $a^{2}=\sigma_{\mathrm{v}}^{2} / R$, versus the molecular gas mass surface density $\Sigma_{\mathrm{H}_{2}}$ for GMCs in M51, NGC6946, NGC628, M31, M33 and the LMC. The solid diagonal line shows the solution for simple gravitational equilibrium. The $\mathrm{V}$-shaped curves represent solutions to Equation 2.1, for different values of the external pressure between $P_{\text {ext }} / k_{B}=10^{3}$ and $10^{7} \mathrm{~K} \mathrm{~cm}^{-3}$. In contrast to the Larson scaling relations, the $\Sigma_{\mathrm{H}_{2}}$ values of GMCs vary by more than two orders of magnitude, and the linewidth-size coefficient increases with increasing $\Sigma_{\mathrm{H}_{2}}$. Right. Median internal pressure of the GMCs versus an estimate of the external pressure within the region of each galaxy covered by the $\mathrm{CO}$ observations. The internal pressures are estimated from GMC properties and the external pressures are estimated using an expression derived by Koyama \& Ostriker (2009) for the total pressure at the disk midplane (see text). The solid diagonal line indicates equality. The vertical error bars indicate the dispersion of the internal pressure estimates for individual GMCs, while the horizontal bars indicate the range of external pressures across the surveyed region.

\section{Physical Properties of Extragalactic GMCs}

From a theoretical perspective, it might be expected that GMC properties depend on their galactic environment (e.g. Elmegreen 1989), but this has been difficult to establish observationally. The first comparative studies of GMC populations in nearby (mostly Local Group) galaxies instead found that the physical properties of extragalactic GMCs are very similar to the properties of GMCs in the inner Milky Way (e.g. Blitz et al. 2007; Bolatto et al. 2008). There are several caveats to the universality of this conclusion, primarily that the first extragalactic surveys could only parameterise the brightest, most massive GMCs in external galaxies, and that the diversity of galactic environments in Local Group galaxies is rather limited. Moreover, a re-analysis of the GMCs in the S87 catalogue has shown that these GMCs are inconsistent with the Larson scaling relations that are regularly used as the basic empirical benchmark of cloud dynamics (Larson 1981) (Heyer et al. 2009). Several recent works targeting individual galaxies have reported variations in the typical mass and dynamical state of GMCs (e.g. Hirota et al. 2011; Utomo et al. 2015), but differences in methodology, as well as the heterogeneity of the original survey datasets, have prevented a conclusive answer emerging from literature measurements.

In the left panel of Figure 1, we plot $a^{2}$ versus $\Sigma$ for the GMC populations in our six galaxies. We emphasize that the datapoints in these plots represent our best effort to compare 'like with like', rather than extracting the most precise information possible from each datacube. The GMC properties are estimated using the same CPROPSTOO algorithms for identifying cloud structures and measuring their properties (Leroy et al. in 
prep), and the algorithms are applied to datacubes that have been brought to the same spectral $\left(5 \mathrm{~km} \mathrm{~s}^{-1}\right)$ and spatial $(53 \mathrm{pc})$ resolution. Note that this can yield quite different results to the properties determined from the original, higher resolution datasets. The sensitivity (per $5 \mathrm{~km} \mathrm{~s}^{-1}$ channel) of the homogenized datasets is $0.5 \mathrm{~K}$ for NGC6946, $\sim 0.2 \mathrm{~K}$ for M51 and M33, and $0.05 \mathrm{~K}$ for the LMC, NGC628 and M31.

If extragalactic GMCs strictly obeyed Larson's scaling relations, the datapoints in the left panel of Figure 1 would cluster around $\Sigma \sim 100 \mathrm{M}_{\odot} \mathrm{pc}^{-2}$, and $a^{2}=\sigma_{\mathrm{v}}^{2} / R=$ $(\pi G \Sigma / 5)$. Instead, $a^{2}$ increases with $\Sigma$ for extragalactic GMCs, both among and within individual galaxies, and that typical mass surface density of GMCs varies between $\sim 10$ and $10^{3} \mathrm{M}_{\odot} \mathrm{pc}^{-2}$. The solid diagonal line in the left panel of Figure 1 represents $a^{2}=$ $(\pi G \Sigma / 5)$, which is where GMCs in simple virial equilibrium should lie. The majority of extragalactic GMCs follow a trend that is roughly parallel to but displaced upwards from the relationship for virialized clouds, i.e. they have an excess of kinetic energy relative to simple virial equilibrium. Following the formulation of Field et al. (2011), the dashed curves in the left panel of Figure 1 indicate the solutions for clouds in a pressure-bounded equilibrium configuration,

$$
a^{2}=\frac{\sigma_{r m v}^{2}}{R}=\frac{1}{3}\left(\pi \Gamma G \Sigma+\frac{4 P_{e x t}}{\Sigma}\right)
$$

for external ISM pressures $P_{\text {ext }} / k_{B}$ between $10^{3}$ and $10^{7} \mathrm{~cm}^{-3} \mathrm{~K}$. $\Gamma$ is a factor that depends on the internal density distribution of a cloud. We adopt $\Gamma=0.6$, corresponding to a cloud with uniform density. The data points do not lie along the dashed curves, which means that the GMCs in each galaxy do not tend towards pressure equilibrium for a single value of $P_{\text {ext }}$. If pressure is important to the dynamical state of clouds, then the large scatter for each GMC population suggests that clouds within a single galaxy experience significant external pressure variations.

Nevertheless, global properties of the host galaxy do seem to influence the average GMC properties. The right panel of Figure 1 shows the average internal pressure of the GMC population in each galaxy versus the total ISM pressure at the disk midplane. We estimate the internal pressure $P_{\text {int }} / k_{B} \sim \rho \sigma_{\mathrm{v}}^{2}=1176\left(\frac{M}{R^{3}}\right) \sigma_{\mathrm{v}}^{2} \mathrm{~cm}^{-3} \mathrm{~K}$, where $M$ is the cloud mass in solar masses, $R$ is the cloud radius in parsecs, and $\sigma_{\mathrm{v}}$ is the cloud velocity dispersion in $\mathrm{kms}^{-1}$. The midplane pressure $P_{\text {mid }}$ for each galaxy is estimated by combining maps of the stellar and gas surface densities and the atomic gas velocity dispersion (each at 500 pc resolution) according to equation 8 of Koyama \& Ostriker (2009). Defined in this way, $P_{m i d}$ approximates the mass-weighted mean pressure (including self-gravity) in the interstellar gas. It is a factor of $\sim 10$ greater than the pressure in the diffuse nonself-gravitating ISM, and a factor of $\sim 4$ greater than the formulation for the external pressure of Blitz \& Rosolowsky (2006) and Elmegreen (1989) that have been used in several previous studies. The vertical error bars in the right panel of Figure 1 reflect the $1 \sigma$ dispersion of the $P_{\text {int }}$ values in each GMC catalogue; the horizontal error bars reflect the range in the per-pixel $P_{m i d}$ values across each survey's field-of-view. For both axes, measurement and systematic uncertainties are not taken into account.

Regardless of the precise formulation for $P_{m i d}$, we obtain the robust result that the typical internal pressure of GMCs is greater in galaxies with higher midplane pressures. More work is required to understand the physical origin of the observed correlation. The average internal pressure of GMCs also increases with quantities such as the stellar surface density $\Sigma_{*}$ and the molecular fraction of the neutral ISM $R_{m o l}=\frac{\Sigma_{\mathrm{H}_{2}}}{\Sigma_{\mathrm{HI}}}$; the current data are insufficient to determine whether the ISM pressure is responsible for the variation in GMC properties, or whether the main driver is a quantity that contributes to $P_{m i d}$. M33 
appears to lie slightly away from the main trend. This may partly be due to the modest signal-to-noise of the BIMA+FCRAO datacube, in which we detect only $\sim 70$ clouds, presumably those with the highest CO surface brightness. A new survey by the IRAM $30 \mathrm{~m}$ telescope has identified > 500 clouds in M33 (Druard et al. 2014), and should yield a more reliable estimate for typical GMC properties in M33.

\section{GMC Mass Distributions in Nearby Galaxies}

The GMC mass distribution describes how a galaxy's molecular gas reservoir is distributed across structures of different mass. In the Milky Way, GMCs follow $d N \propto$ $M^{\gamma} d M$, with $\gamma \sim-1.5$ in the inner disk (S87) and $\gamma \sim-1.8$ in the outer disk (Heyer, Carpenter \& Snell 2001). In star formation theory and numerical modelling, a value of $\gamma \sim-1.5$ has been used as both a benchmark (e.g. Wada, Spaans \& Kim 2000) and input (e.g. Krumholz \& McKee 2005), although the universality of this $\gamma$ value has not been empirically verified. New surveys of CO emission in M51 (Colombo et al. 2014) and M33 (Gratier et al. 2012) instead suggest that the shape of the GMC mass distribution varies with galactocentric radius and kinematic environment, although the physical agents that determine the shape of the distribution are yet to be securely identified.

While GMC mass distributions in several nearby galaxies have now been studied (e.g. Rosolowsky 2005; Fukui et al. 2008; Gratier et al. 2012; Colombo et al. 2014), a direct comparison of these results is not straightforward since the shape of the mass distribution depends on the resolution and sensitivity of the input datasets, as well as the decomposition algorithm and the completeness limit (e.g. Reid et al. 2010). In Figure 2, we show the cumulative mass distributions for the GMC populations of our six galaxies, using the same GMC catalogues as in Section 2. Strictly, the curves in Figure 2 represent the CO luminosity function of each GMC population. We convert the CO luminosity to mass using the simple scaling $M_{G M C}=\alpha_{\mathrm{CO}} L_{\mathrm{CO}}$, assuming a constant $\alpha_{C O}=4.4 \mathrm{M}_{\odot}\left(\mathrm{K} \mathrm{km} \mathrm{s}^{-1} \mathrm{pc}^{2}\right)^{-1}$ for all galaxies. This is equivalent to $\mathrm{X}_{\mathrm{CO}, \mathrm{MW}}=2 \times 10^{20} \mathrm{~cm}^{-2}\left(\mathrm{~K} \mathrm{~km} \mathrm{~s}^{-1}\right)^{-1}$, and includes the mass contribution of helium. In the left panel, the curves are normalized by the deprojected area that was surveyed in each galaxy. In this case the vertical offsets between the curves reflect a combination of the different sensitivity limits of the input CO surveys and genuine physical variations in the number surface density of GMCs. The surveys of LMC, M31 and NGC628 have roughly equivalent sensitivity, so the relative amplitude of their mass distributions shows that NGC628 is much richer in molecular clouds than the surveyed regions of M31 and the LMC. M51 is likewise much more GMC-rich than M33.

In the right panel of Figure 2, the curves are normalized by the total number of clouds. This normalization is useful to compare the shape of the mass distribution in the different galaxies. Overall, the mass distribution shapes are quite similar, although the mass distribution continues to a much higher maximum cloud mass in M51 and NGC6946 than in the other galaxies. Across the mass range where the distributions are well-represented by a power-law, the distribution slope is also flatter (by $\sim 0.5 \mathrm{dex}$ ) in M51 and NGC6946. Following Rosolowsky (2005), we model the cumulative GMC mass distribution as a truncated power-law with the form

$$
N\left(M^{\prime}>M\right)=N_{0}\left[\left(\frac{M}{M_{0}}\right)^{\gamma+1}-1\right],
$$

where $\gamma$ is the slope of the differential mass spectrum, $M_{0}$ is the maximum mass in the distribution, and $N_{0}$ is the number of clouds more massive than the mass limit where 

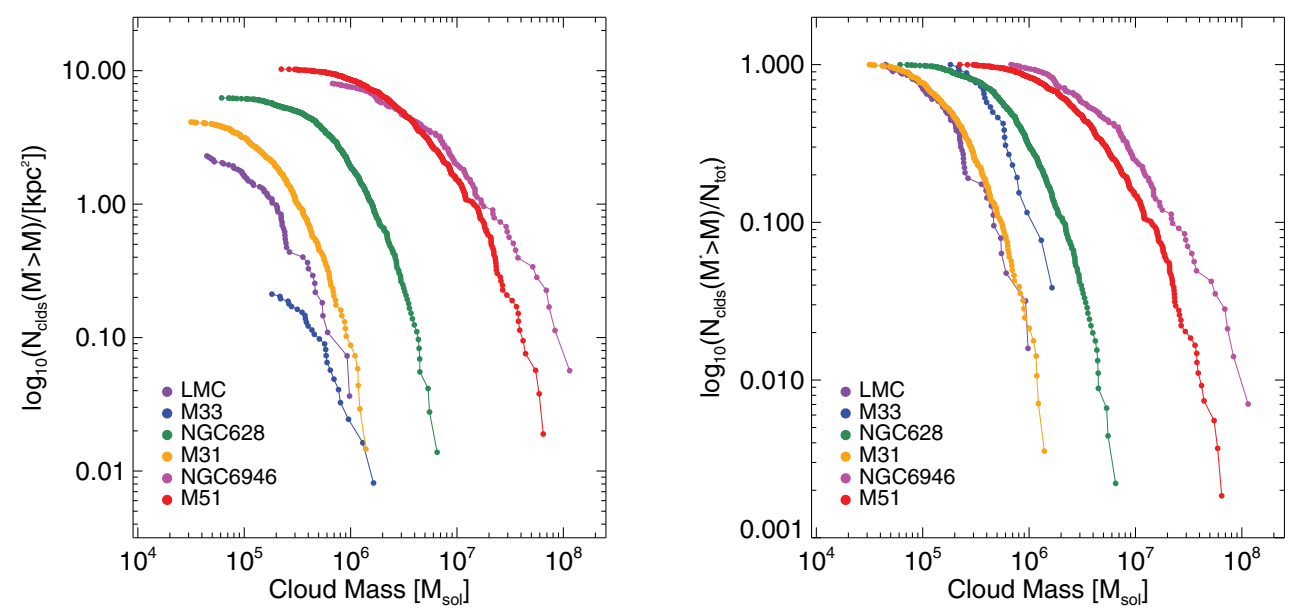

Figure 2. Cumulative mass distributions of GMCs in M51, NGC6946, NGC628, M31, M33 and the LMC, normalized by the area of the CO survey (left panel) and by the total number of identified clouds (right). The vertical offset between the curves in the left panel reflects variations in the number density of GMCs. The right panel shows that the slope of the GMC mass distribution is flatter in the denser, more massive systems (M51, NGC6946).

the mass distribution deviates from a simple power-law. A truncated power-law provides a better description of the mass distribution than a simple power-law for most of our galaxies. The exceptions are M33 and the LMC, where relatively few clouds are identified and a simple power-law fit cannot be excluded. As noted by previous studies, we find that the best-fitting values of $\gamma, N_{0}$ and $M_{0}$ are sensitive to the lower mass limit that we include in the fit. If we restrict the fit to clouds with masses above the median GMC mass, we find $\gamma \sim-1.8$ for M51 and NGC6946, $\gamma \sim-2.2$ for M31 and NGC628, and $\gamma<-2.5$ for M33 and the LMC. Using a lower mass limit of only 5 times the RMS sensitivity of each survey decreases the best-fitting values of $\gamma$ by $\sim 0.4$ dex, but the general trend among galaxies is preserved. If the GMC mass distribution in M33 follows a truncated power-law, then the steep slope that we derive may stem from the modest sensitivity of the BIMA+FCRAO survey.

Is the shape of the GMC mass distribution universal, or does it depend on properties of the host galaxy? While more work is required to identify the physical mechanisms that regulate the growth and destruction of GMCs, our analysis tends to favour the idea that the shape GMC mass distribution depends on galactic environment. In Figure 3, we plot the slope of the GMC mass distribution versus several basic quantities that characterize the local physical conditions: $\Sigma_{*}$ (left), $\Sigma_{\text {gas }}=\Sigma_{\mathrm{HI}}+\Sigma_{\mathrm{H}_{2}}$ (middle) and $R_{\text {mol }}$ (right). These are empirical estimates measured on $500 \mathrm{pc}$ scales. These plots show that the slope of the GMC mass distribution tends to flatten as the galactic environment becomes denser and more molecule-rich. The observed trends roughly follow $\gamma \propto \Sigma_{*}^{0.6}$, $\gamma \propto \Sigma_{\text {gas }}^{0.8}$, and $\gamma \propto 0.2 R_{\text {mol }}$, but the data are insufficient to characterize the trends very precisely. Qualitatively similar trends are observed for the upper $10^{\text {th }}$ percentile GMC mass $M_{10}$ and the number surface density of GMCs $\mathcal{N}$, but the absolute values of $M_{10}$ and $\mathcal{N}$ are dependent on the sensitivity of the input datasets. A survey of a diverse sample of nearby galaxies with uniform sensitivity and spatial resolution would place these results on a more quantitative footing. 

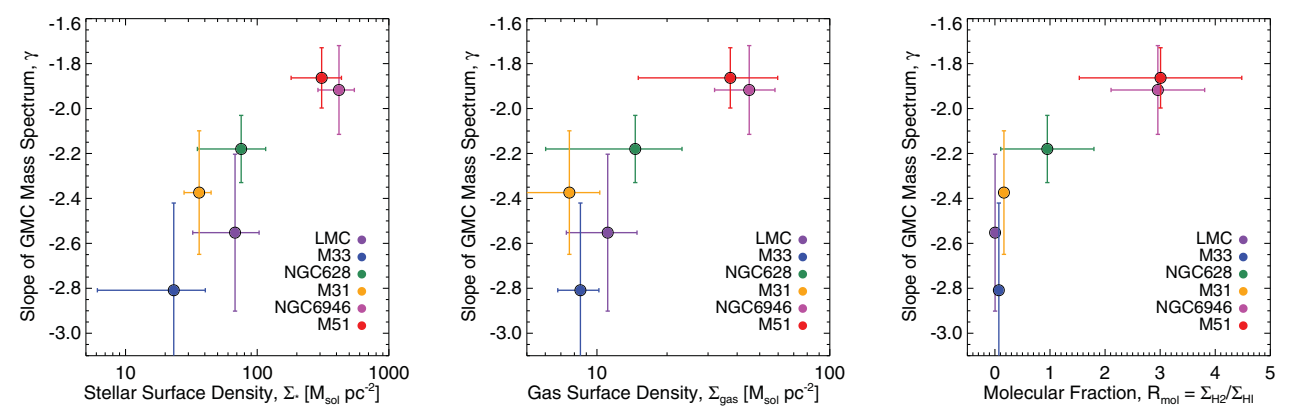

Figure 3. The power-law slope of the GMC mass distribution versus the local stellar surface density $\Sigma_{*}$ (left), neutral gas surface density $\Sigma_{\mathrm{gas}}=\Sigma_{\mathrm{HI}}+\Sigma_{\mathrm{H}_{2}}$ (middle), and molecular fraction $R_{m o l}=\Sigma_{\mathrm{H}_{2}} / \Sigma_{\mathrm{HI}}$. The quantities on the x-axis are measured on $500 \mathrm{pc}$ scales. The vertical error bars represent the uncertainty in the fit. The horizontal error bars indicate the $1 \sigma$ dispersion of values within the area of the CO surveys. Taken together, the plots show that the slope of the GMC mass distribution tends to flatten as the galactic environment becomes denser and more molecule-rich.

\section{GMC Lifetimes: An Observational Estimate in M51}

Resolved observations of GMC populations in nearby galaxies present a new opportunity to place empirical constraints on the timescale of GMC evolution. This timescale is important for star formation theory, as it sets a natural limit to the timescale over which gas can be transformed into stars. Previous efforts to reconstruct GMC lifetimes in external galaxies have used the association between GMCs and young stellar phenomena with better known evolutionary timescales, e.g. HII regions and young stellar clusters, to estimate the lifetime of the associated GMC (e.g. Leisawitz et al. 1989). In M33 and the Large Magellanic Cloud, this approach yields a typical GMC lifetime of $\sim 30$ Myr (e.g. Kawamura et al. 2009; Miura et al. 2012). The universality of this result - i.e. whether star-forming clouds in different galactic environment evolve over a similar timescale is uncertain. Using the PAWS dataset for M51, we have developed a new method for estimating GMC lifetimes in disk galaxies that is independent of their relationship with young stellar phenomena (Meidt et al. 2015). The method requires wide-field, sensitive observations that capture a significant number of clouds (more than a few hundred), as well as detailed knowledge of gas dynamics in the galactic disk. The basic premise is that a short cloud lifetime (i.e. less than a galactic orbital period) should manifest itself as a decrease in the number of clouds across the inter-arm region between strong spiral arms. The observed decrease in cloud numbers constrains the ratio between the cloud lifetime and the travel time between the arms.

More formally, for an initial population of clouds $N_{0}$ at time $t_{0}$, we can estimate the characteristic cloud lifetime $\tau$ by measuring the cloud population $N_{1}$ at a later time $t_{1}$. Assuming the time evolution of GMCs is dominated by their destruction, then the cloud population will be reduced to zero after $\tau$, i.e.

$$
N_{0}-\frac{N_{0}-N_{1}}{t_{1}-t_{0}} \tau=0 .
$$

In this equation, the rate $-\frac{\left(N_{0}-N_{1}\right)}{\left(t_{1}-t_{0}\right)}$ represents the effective rate of change in the GMC population (i.e. the net result of both formation and destruction processes), assuming that the rates of cloud formation and destruction remain constant with time. When the 
cloud formation rate is low,

$$
\frac{N_{0}-N_{1}}{t_{1}-t_{0}}=N_{0}\left(\frac{1}{\tau_{\text {dest }}}-\frac{1}{\tau_{\text {grow }}}\right) \approx N_{0}\left(\frac{1}{\tau}\right) .
$$

In this equation, $\tau_{\text {dest }}$ is the required to reduce the initial cloud population to zero, and $\tau_{\text {grow }}$ is the time required to increase the initial population by $N_{0}$.

Estimating $\tau$ from the observed spatial distribution of GMCs therefore involves measurements of $N_{1}$ and $N_{0}$ for any two adjacent regions between which there is a well-defined travel time $t_{1}-t_{0}$. Counting the number of clouds at different spatial locations along a common trajectory serves as a proxy for the time evolution of a individual cloud. The method is well-suited to the interarm region of disk galaxies chiefly because: (1) in contrast to the spiral arms where there are significant non-circular motions, interarm clouds follow a roughly circular path and their angular velocity $V_{\phi}$ can be approximated by the circular velocity in the disk; and (2) cloud formation mechanisms are less efficient in the interarm region, hence $\tau$ is likely to provide a more direct measure of the true cloud destruction timescale $\tau_{\text {dest }}$. With sufficient cloud numbers, multiple interarm zones could be considered, yielding several independent measures of $\tau$. In practice, we apply the method to the PAWS observations of M51 by dividing the interarm into two halves (zone I and zone II) spanning the entire interarm region. In this case, counting the cloud numbers in the two zones $N_{0}=N_{I}$ and $N_{1}=N_{I I}$ is equivalent to the time for an individual cloud to cross half the interarm distance $t_{\text {travel }} / 2$, where $t_{\text {travel }}$ is the full interarm distance divided by the angular velocity $V_{\phi}$. Equation 4.1 can then be rewritten

$$
\tau=\frac{t_{\text {travel }}}{2} \frac{N_{I}}{N_{I}-N_{I I}} .
$$

In M51, we have applied our method to six radial bins covering the interarm zone between galactocentric radii $R=1.3$ and $R=3.3 \mathrm{kpc}$. Using the rotation curve model derived by Meidt et al. (2013) to estimate the circular velocity, the full interarm travel time $t_{\text {travel }}$ across this radial range varies between 25 and $50 \mathrm{Myr}$. On average, we find that the cloud population decreases by $\sim 80 \%$ from zone I to zone II, suggesting that a characteristic cloud lifetime that is shorter than $t_{\text {travel }}$. Inside $R \sim 2.5 \mathrm{kpc}$, we find $\tau \sim 20$ to $30 \mathrm{Myr}$. This lifetime only applies to clouds with masses above the PAWS completeness limit, i.e. $M>$ a few times $10^{5} \mathrm{M}_{\odot}$. The radial variation in the ratio $\frac{N_{I}}{N_{I}-N_{I I}}$ is modest. This is inconsistent with a constant cloud lifetime since $t_{\text {travel }}$ increases with galactocentric radius. The observed trend is qualitatively consistent with shear being the primary agent of cloud destruction in the interarm of M51's inner disk, since shear weakens with galactocentric radius. Quantitatively, our estimated cloud lifetimes are in good agreement with the shear timescale (as measured from the background Oort A) for $R<2.5 \mathrm{kpc}$. At larger radii, however, this good agreement breaks down. Maps of highmass star formation tracers in M51 indicate that the presence of numerous giant HII regions at $R \sim 2.5 \mathrm{kpc}$ (e.g. Meidt et al. 2013, Schinnerer et al. 2013). Cloud destruction in these radial bins may therefore be governed by the interplay of shear and star formation feedback, rather than shear alone.

\section{Summary and Outlook}

The next decade promises to be a productive moment for connecting the cloud- and galactic-scale views of star formation. The first systematic comparisons of extragalactic GMC properties contained only $\sim 100$ clouds in total, mostly in the Local Group dwarfs; facilities such as ALMA and NOEMA will resolve thousands of GMCs across a diversity 
of systems at much larger distances. The results highlighted in this contribution provide an initial look at how galactic environment influences both the overall structure of the molecular ISM in galaxies, and the dynamical state and evolutionary timescale of individual clouds. With a new generation of extragalactic datasets that can be directly compared to cloud-scale models of star formation, we can expect good progress in understanding how galaxies regulate their gas density distribution, and how the physics of star formation and feedback on cloud scales leads to the observed global pattern of star formation in galaxies.

\section{References}

Blitz, L. \& Rosolowsky, E. 2006, ApJ 650, 933

Blitz, L., Fukui, Y., Kawamura, A. et al. 2006, in: B. Reipurth, D. Jewitt, \& K. Keil (eds.), Protostars and Planets $V$ (Tucson: University of Arizona Press), p. 81

Bolatto, A., Leroy, A., Rosolowsky, E. et al. 2008, ApJ, 686, 948

Colombo, D., Hughes, A., Schinnerer, E. et al. 2014, ApJ 784, id. 3

Donovan Meyer, J., Koda, J., Momose, R. et al. 2013, ApJ, 772, id. 107

Druard, C., Braine, J., Schuster, K. et al. 2014, A\&A 567, id. 118

Elmegreen, B. 1989, ApJ, 338, 178

Field, G., Blackman, E., \& Keto, E. 2011, MNRAS, 416, 710

Fukui, Y., Kawamura, A., Minamidani, T. et al. 2008, ApJS, 178, 56

Gratier, P., Braine, J., Rodriguez-Fernandez, N. et al. 2012, A\&A 542 , id. 108

Heyer, M., Carpenter, J., \& Snell, R. 2001, ApJ, 551, 852

Heyer, M., Krawczyk, C., Duval, J. et al. 2009, ApJ, 699, 1092

Hirota, A., Kuno, N., Sato, N., Nakanishi, H. et al. 2011, ApJ, 737, id. 40

Hughes, A., Meidt, S., Colombo, D. et al. 2013, ApJ, 779, id. 46

Kawamura, A., Mizuno, Y., Minamidani, T. et al. 2009, ApJS, 184, 1

Koyama, H. \& Ostriker, E. 2009, ApJ, 693, 1346

Krumholz, M. \& McKee, C. 2005, ApJ, 630, 250

Larson, R. 1981, MNRAS, 194, 809

Leisawitz, D., Bash, F., \& Thaddeus, P. 1989, ApJS, 70, 731

Meidt, S., Hughes, A., Dobbs, C. et al. 2015, ApJ, 779, id. 45

Meidt, S., Schinnerer, E., García-Burillo, S. et al. 2013, ApJ, 779, id. 45

Miura, R., Kohno, K., Tosaki, T. et al. 2012, ApJ, 761, id. 37

Pety, J., Schinnerer, E., Leroy, A. et al. 2013, ApJ 779, id. 43

Reid, M.., Wadsley, J., Petitclerc, N. et al. 2010, ApJ, 719, 561

Rosolowsky, E 2005, PASP, 117, 1403

Rosolowsky, E., Keto, E., Matsushita, S. et al. 2007, ApJ 661, 830

Schinnerer, E., Meidt, S., Pety, J. et al. 2013, ApJ, 779, id. 42

Solomon, P., Rivolo, A., Barrett, J. et al. 1987, ApJ, 319, 730

Utomo, D., Blitz, L., Davis, T. et al. 2015, ApJ, 803, id. 16

Wada, K., Spaans, M., \& Kim, S. 2000, ApJ, 540, 797

Wong, T., Hughes, A., Ott, J. et al. 2011, ApJS, 197, id. 16 